\title{
Focus Point on Systems and Security: Advanced Methods with Chaos and Complexity
}

Published online: 9 August 2018 - C) Società Italiana di Fisica / Springer-Verlag GmbH Germany, part of Springer Nature, 2018

During the last two decades, chaos theory has been applied in the field of secure communications, since the system is a source of pseudo random generator, and can be applied as a cryptosystem due to the synchronization phenomenon. On the other hand, dynamical complexity in information theory plays an effective role in extracting information inside the dynamics. Several entropy measures, such as approximate entropy, sample entropy, fuzzy recurrence entropy, etc., have been developed to quantify the complexity of time series arising from chaotic models and stochastic processes, such as Markov chains.

Complexity may arise in various nonlinear real-world phenomena. For example, the human heart is a complex system, since it has many interacting subunits to keep it active. A congestive failure heart is less complex compared to a normal healthy heart, since it fails to work properly with many interacting subunits. Another popular example are communication networks (such as the Internet, which is a complex model), where complexity varies with different parameters, such as structure and evolution of the network, connection and node diversity, etc.

As of late, it has been investigated that, in the case of optical communications (semiconductor lasers), complexity plays an important role in investigating the dynamics, instabilities, securities and output power, as it is strongly correlated with the laser intensities. So, dynamical complexity is also an important tool to investigate the associated security in chaos-based communications.

The aim of the Focus Point on Systems and Security: Advanced Methods with Chaos and Complexity is to explore the trendand the developments of the topic and its possible applications to science and engineering. A collection of seven high-quality regular articles has been included on several complexity measures of discrete and continuous systems and time series, analyses on optical networks and complex dynamical networks, etc. Applications on audio, video and image encryptions have also been reported with integer- and fractional-order chaotic systems. The contributions may be effective to the researchers, scientists and engineers in the subject area, as they contain novel analyses with real-world implementations.

I would like to thank the authors for their contributions and the referees for their efforts in reviewing the manuscripts. Finally, I would like to thank all members of EPJ Plus, for hosting this Focus Point and their continuous support. 